\title{
On Air-to-Water Radio Communication between UAVs and Water Sensor Networks
}

\author{
Jacob Palmer ${ }^{1}$, Nicholas Yuen ${ }^{2}$, John-Paul Ore ${ }^{3}$, Carrick Detweiler ${ }^{3}$, and Elizabeth Basha ${ }^{1}$
}

\begin{abstract}
Ocean monitoring using underwater sensor networks faces communication challenges in retrieving data, communicating large amounts of data between nodes, and covering increasing spatial regions while remaining connected. With underwater sensor networks that are capable of surfacing, unmanned aerial vehicles (UAVs) provide a solution to this by providing radio-based data muling services, but, as this area is still unexplored, the utility of this solution is unclear. In this paper, we examine the theoretical expectations, perform several field experiments, and analyze the communication success rates of 802.15.4 radios near the water surface both communicating between surface nodes as well as between a node and the UAV. These indicate that on the water surface internode radio communication is poor, but node to UAV communication can provide both reasonable ranges and success rates. We additionally measure and analyze the energy aspects of the systems, determining the impacts of parameters such as network size and distance between nodes on the UAV energy. Finally, we consolidate the information into an algorithm outlining how to configure and design hybrid UAV and underwater sensor network systems.
\end{abstract}

\section{INTRODUCTION}

Ocean monitoring using sensor networks provides daunting technical challenges and promising scientific opportunities. The challenges in collecting data from underwater sensors include low communication bandwidth, slow and power-hungry acoustic modems, and deep and physically inaccessible nodes. Addressing these challenges requires communication improvements and automated system access.

In this paper, we propose a system consisting of both underwater sensor nodes and an unmanned aerial vehicle (UAV), where the underwater nodes can surface to communicate by radio with the UAV. The UAV helps the underwater nodes overcome poor node-to-node signal propagation near the water surface due to the antennas' proximity to the water. By using a UAV, we gain a significantly increased radio range from the node at the surface to the UAV in the air. This allows us to leverage radios that are orders of magnitude faster, use less energy, and cost significantly less than acoustic modems.

\footnotetext{
${ }^{1}$ E. Basha and J. Palmer are with the Department of Electrical and Computer Engineering, University of the Pacific, Stockton, CA 95211, USA ebasha, jpalmer at pacific.edu

${ }^{2} \mathrm{~N}$. Yuen was with the Department of Electrical and Computer Engineering, University of the Pacific, Stockton, CA 95211, USA during this work and is now with NVIDIA. nyuen at pacific.edu

${ }^{3} \mathrm{C}$. Detweiler and J-P Ore are with the Department of Computer Science and Engineering, University of Nebraska-Lincoln, Lincoln, NE 68588, USA carrick, jore at cse.unl.edu

This work was partially supported by NSF CSR-1217428, IIS-1116221, CSR-1217400, and USDA \#2013-67021-20947. Any opinions, findings, and conclusions or recommendations expressed in this material are those of the authors and do not necessarily reflect the views of these agencies.
}

This paper explores the opportunities and limitations of a system with underwater nodes and UAVs, examines theoretical models to determine feasibility, characterizes the packet-success-rate of UAVs over water in field experiments, analyzes the behavior of UAVs based on the data, and defines policies for the use of UAVs with underwater sensor networks. We present a theoretical model of radio propagation over water that characterizes the significantly greater range from water-to-air than from water-to-water.

We then confirm these theories with field experiments that characterize the communication behaviors between the UAV and a surfaced sensor node as well as between two surfaced sensor nodes. With our off-the-shelf 802.15.4 radios we show that surfaced sensor nodes can only communicate 34 meters with $40 \%$ packet-success, while the UAV can communicate with a surfaced node to a range of 212 meters with $75 \%$ packet-success. While these results are specific to the radios we use, we believe the significant disparity between the types of communication will generalize to other radios near water. These results identify limitations in using radios for surface communication between nodes and opportunities for using UAVs as data mules.

We also experimentally measure energy characteristics of the systems to understand the constraints on system lifetime and use this information to model the energy in the system. With a clear understanding of the actual communication and energy behaviors of the system, we analyze the options and tradeoffs in using the sensor network with the UAV. Specifically, we develop an algorithm that determines the network size that a single UAV can support given constraints. Our system must ensure that the UAV can travel to the network, collect required data from the network, and safely return to the base with the collected information. The algorithm takes into account UAV and communication energy and allows us to define the distance of the network, the amount of data to collect, and the node spacing. This provides a general framework to determine when it is appropriate to deploy UAV-aided water sensor networks.

This paper is organized as follows. Section II outlines related work. Section III briefly describes the underwater sensor network and UAV. Section IV discusses the theory relating expected signal quality to height and distance from the nodes. Section V then discusses communication aspects of the systems, describing the field experiments to characterize the system and the result analysis. Section VI addresses the energy aspects while Section VII outlines combined energycommunication analyses and algorithms using these results. We discuss our conclusions and future work in Section VIII. 


\section{RELATED WORK}

Prior research has not explored the use of UAVs as data mules with underwater sensor networks, to the best of our knowledge. In this section we summarize work related to using UAVs as data mules for land based sensor networks and AUVs as data mules for underwater sensor networks.

Teh et al. presented a preliminary design for land based UAV data muling using a modified Boomerang 60 model aircraft and Fleck 3 sensor nodes [1]. They performed three major experiments: (1) testing the radio communication range between ground and UAV, (2) demonstrating the data muling capability of the UAV, and (3) integrating the Fleck 3 node with the UAV autopilot. Overall, the paper concluded that UAVs are feasible data mules for wireless sensor networks.

Isaacs et al. combined a Procerus Unicorn UAV with time-of-arrival sensors; their focus is on event localization and path planning for the UAV to efficiently collect the data from the nodes [2]. Klein et al. extended this work to more extensive path planning and localization [3]. Todd et al. use an UAV to power and collect data from nodes performing structural health monitoring [4]. Sugihara and Gupta developed several algorithms based on an UAV data mule where the work by Todd et al. as is their application case study [5], [6], [7], [8]. These works focus more on path planning and ground communication, while our work focuses more on determining what sensor network system the UAV can support and water communication.

Vasilescu et al. presented AUV data muling using the Amour AUV, Starbug AUV, and the AquaFleck underwater sensor network platform [9]. This paper found that an AUV performing data muling with an optical modem provided a power efficient, effective means of harvesting data in underwater sensor networks. Doniec et al. further explored optical AUV data muling [10]; they focused primarily on novel AUV and optical communication design. Li et al. formulated path planning algorithms for an AUV operating as an underwater data mule [11] and supports the need for data muling with water-based sensor networks. Our paper focuses on enabling and scaling communication between underwater sensors that can surface and use radio to communicate with UAVs.

\section{SySTEM OVERVIEW}

In prior work, we developed the AquaNode platform [12], which is an underwater sensor network node with depth adjustment capabilities and is shown in Figure 1. It is anchored in place and can adjust its depth to surface for radio communication or submerge to obtain sensor readings from the full water column. While underwater, it can communicate with neighbors using acoustic communication, although the number of bits that can be sent is limited and in shallow water conditions (such as rivers) acoustic communication can be extremely challenging. See [12] for additional details on the depth adjustment system and communication systems.

For our aerial vehicle we use an Ascending Technologies Hummingbird quad-copter. It has payload capabilities of 200g, a flight time of approximately 20 minutes, and can

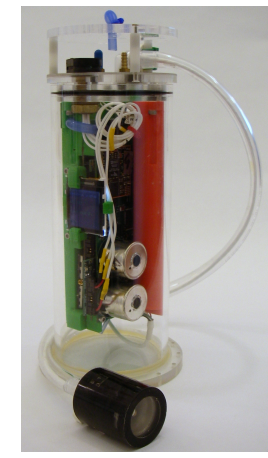

Fig. 1. AquaNode Platform.

fly at over $14 \mathrm{~m} / \mathrm{s}$. Outdoors, the Hummingbird uses GPS to maintain position and a pressure sensor altimeter for height control. This UAV can be controlled by a remote ground station via 802.15.4 radio or by using a small onboard computer for full autonomy. In this experiment, we controlled it remotely and added a secondary 802.15.4 radio on a nonconflicting channel to communicate with the nodes.

\section{Radio Propagation OVER WATER TheORY}

To better understand expected areas of best signal strength, we constructed a theoretical model based on existing research [13], [14], [15], [16]. The model uses a two-ray approach that incorporates the radiation pattern of the antenna, attenuation caused by reflecting off the water surface, and free space attenuation along both paths. The ratio between the line of sight (LOS) path and the reflected path determines the final assessment of predicted signal quality. Areas where the LOS attenuation surpasses the minimum signal strength required by the receiver are poor quality areas regardless of the LOS to reflection ratio.

For the 1/4 wavelength dipole antennas we use, the radiation pattern function is:

$$
F=\frac{\cos (\pi L \cos \theta)-\cos (\pi L)}{\sin \theta}
$$

where $\mathrm{L}$ is the ratio of the antenna's length to the wavelength and theta is the angle with respect to the vertical direction about the center of the antenna. This yields a toroidal pattern. The present model assumes an isotropic receiving antenna.

Jiang et al.[13] approximated the frequency dependent dielectric permittivity $\bar{\varepsilon}_{r}(f)$ of fresh water using the Debye model:

$$
\bar{\varepsilon}_{r}(f)=\varepsilon_{\infty}+\left(\frac{\varepsilon_{s}-\varepsilon_{\infty}}{1+\left(i \frac{f}{f_{\text {ref }}}\right)}\right)-\frac{i \sigma}{2 \pi f \varepsilon_{0}}
$$

where $\varepsilon_{s}=80 \mathrm{Fm}^{-1}$ is the real relative permittivity at low frequencies, $\varepsilon_{\infty}=4.22 \mathrm{Fm}^{-1}$ is the real relative permittivity at high frequencies, $f_{\text {ref }}=17.4 \mathrm{GHz}$ is the relaxation frequency, and $\sigma=0.01 \mathrm{Sm}^{-1}$ is the estimated conductivity of freshwater. Hollister [15] described the vertically polarized reflection coefficient $r_{v}$ as the real portion of:

$$
r_{v}=R e\left\{\frac{\bar{\varepsilon}_{r}\left(h_{t}+h_{r}\right)-\sqrt{d^{2}\left(\bar{\varepsilon}_{r}-1\right)+\left(h_{t}+h_{r}\right)^{2} \bar{\varepsilon}_{r}}}{\bar{\varepsilon}_{r}\left(h_{t}+h_{r}\right)+\sqrt{d^{2}\left(\bar{\varepsilon}_{r}-1\right)+\left(h_{t}+h_{r}\right)^{2} \bar{\varepsilon}_{r}}}\right\}
$$

where $h_{t}$ is the transmitter height, $h_{r}$ is the receiver height, and $d$ is the distance. The horizontal polarization coefficient 


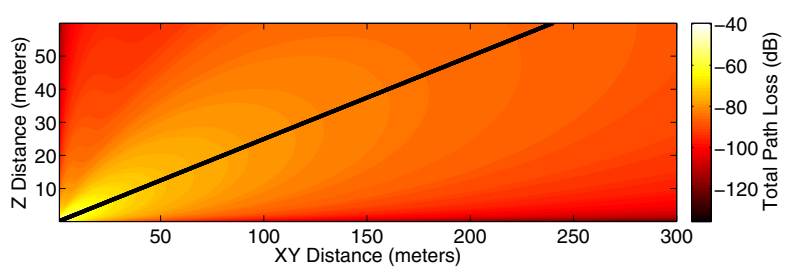

Fig. 2. Superposition of LOS and Reflected Wave Paths.

does not need to be calculated because the antennas being used are vertically polarized. The height of the transmitting antenna was fixed at $4 \mathrm{~cm}$, as this is the height of the sensor node antenna above the surface of the water. Using these equations and trigonometry, we calculate the LOS and reflected paths from the surfaced node to the UAV. Finally, we apply Friis free space model with unity gain along the length of each path.

The phase difference between the LOS and reflected paths is given by:

$$
\theta_{\Delta}=\frac{2 \pi\left(D_{r e f}-D_{L O S}\right)}{\lambda}
$$

The attenuation at any point in two dimensional space away from the node is given by the sum of the LOS and reflected attenuations:

$$
\begin{gathered}
\alpha_{L O S}=10 \log \left(R_{L O S}\left(\frac{\lambda}{4 \pi D_{L O S}}\right)^{2}\right) \\
\alpha_{r e f}=10 \log \left(R_{r e f}\left(\left(1+r_{v}\right) \frac{D_{L O S}}{D_{r e f}} \cos \theta_{\Delta}\right)^{2}\right) \\
\alpha_{\text {total }}=\alpha_{L O S}+\alpha_{r e f}
\end{gathered}
$$

where $R_{L O S}$ and $R_{\text {ref }}$ are the ratios given by the normalized radiation pattern for each respective path.

Figure 2 depicts this attenuation. We see high attenuation close to the water suggesting that we will have limited communication range between nodes on the surface.

\section{COMmunication Field EXPERIMENTS}

The theoretical model provides a first pass estimate of the signal propagation, but does not take into account many of the real-world factors that impact the signal (e.g. waves). In this section we verify our model with in-situ tests. We perform two field experiments to measure communication success rates: (1) between the UAV and a surfaced sensor node and (2) between two surfaced underwater sensor nodes. For both experiments, we define success as instances where both systems communicated bidirectionally. After performing the experiments, we analyze the combined results.

\section{A. UAV to Water Node Radio Communication}

Our first experiment examines ranges and success rates between a node on the water surface and the UAV.

1) Test Configuration: We performed this field experiment on the Platte River, Nebraska, USA; Figure 3 shows the experiment configuration. The static node simplifies the AquaNode platform to just have a 802.15.4 radio and float on the water surface without the depth adjustment system. We placed this node at location "A" seen in Figure 3 and tethered it to the bridge so that it would not move significantly.
For the UAV, we manually flew it at different heights and distances. Nodes "B" through " $\mathrm{N}$ " in Figure 3 outline the locations at which we varied the altitude. We started by manually flying away from the static node at fixed intervals along the bridge. At each interval location, we steadily decreased the altitude until close to the surface, increased the altitude until we noticed a significant decrease in the success rate, and then returned to an altitude level with the bridge. We continued this pattern at increasing distances away from the static node until we reached a point at which we saw significant packet loss while at bridge altitude level. After reaching this point, we moved back towards the static node and repeated the pattern to gain additional data points.

Throughout the experiment, we recorded the GPS location of the UAV, the packets sent from the static node, the packets received at the $\mathrm{UAV}$, and the packets received at a secondary node used for confirming behaviors.

2) Test Results: Figures 4 and 5 depict the results of the experiment. Figure 4 indicates the locations where the UAV did and did not receive packets. The x-axis is the horizontal distance between the UAV and the static water node; this is the Euclidean distance in the XY plane as the UAV had some minor oscillations in y positioning due to manual flying and wind. The $y$-axis is the altitude of the UAV above the river surface. Blue circles indicate received locations while red triangles indicate that the UAV did not receive any packets. From this figure, we can see a clear region of success.

To better understand this region, we can examine Figure 5, which shows the average packet-success rate of radio communication between the UAV and the water node at different vertical and horizontal distances. In the figure, white-yellow colors signify high success rates and black colors signify low to zero success rates. As this figure demonstrates, near the surface of the water at altitudes under 5 meters, the systems have a limited horizontal communication range. Successful communication occurs above 5 meters until the UAV approaches 43 meters above the water surface at which point the packet-success rate drops off sharply. Horizontally, we still see communication until after 249 meters at an altitude of 32 meters; in this location we still see $20 \%$ success rates. Overall, our system achieved the peak packet-success rate of $94 \%$ at approximately 174 meters horizontally at 38 meters above the surface of the water.

We can compare this to our theoretical results from Sec-

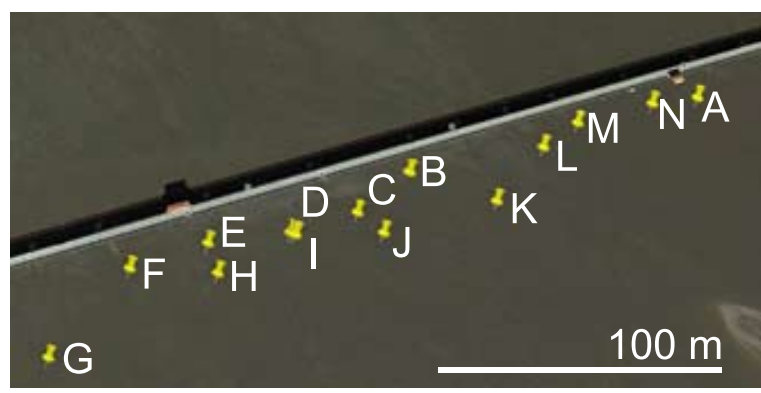

Fig. 3. UAV to water node test configuration. The "A" marker represents the location of the static water node and rest represent the main locations of the UAV. Figure courtesy of Google Earth. 


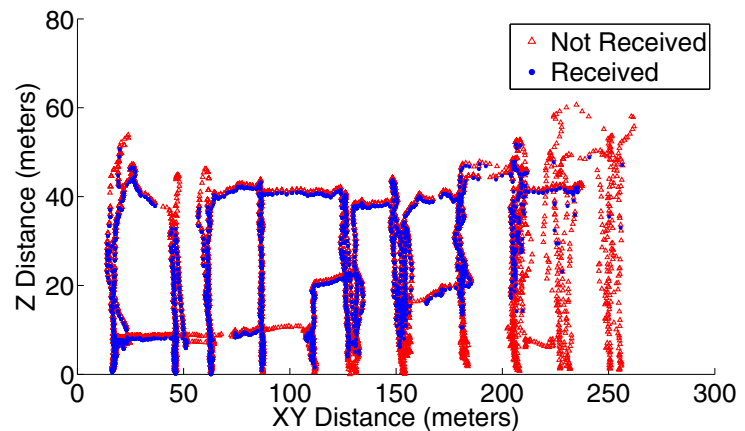

Fig. 4. Radio sent and received messages between water node and UAV at different locations.

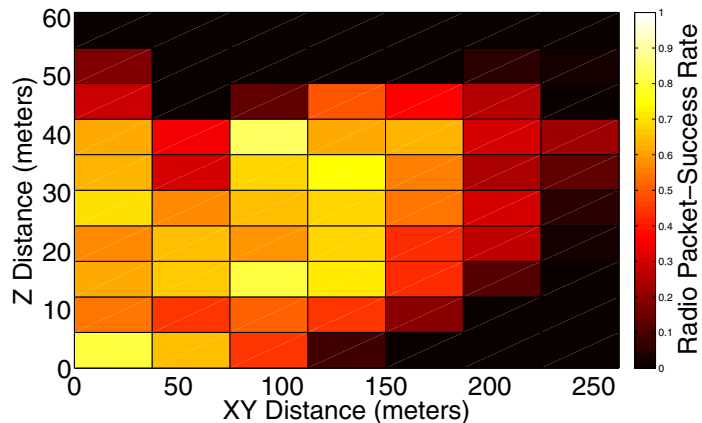

Fig. 5. Radio communication packet-success rates between water node and UAV at different distances.

tion IV. Because the experiment only measured at discrete $\mathrm{XY}$ intervals and, therefore, provided a discrete data set, we had to bin the data in order to extract the angle of the line of least attenuation. For each XY distance bin, we computed the angle of incidence to the $\mathrm{Z}$ altitude bin at which the systems had the best packet reception. After computing angles for all XY distance bins, we compute the average angle. To ensure our results are not overly skewed by the effects of binning, we explored a range of different bin numbers, splitting the $\mathrm{XY}$ distance into 7 to 10 divisions and the $\mathrm{Z}$ altitude into 10 to 15 divisions. For all the possible combinations, this process yielded a range of approximately $11^{\circ}$ to $17^{\circ}$. In Figure 2, the line from this angle of incidence is drawn and is extremely close to $12^{\circ}$, which is the estimated angle of incidence from the model using the same binning method. Real conductivity of the water, waves on the surface, and swaying of the node caused by river currents may all contribute to this difference.

\section{B. Water Node to Water Node Radio Communication}

Our second field experiment determines the communication ranges and success rates between two surfaced nodes communicating via radio.

1) Test Configuration: For this field experiment, we had two identical water nodes that floated on the surface. The first node remained static at position "A" depicted in Figure 6 while the second node moved from positions "B,"'C," and "D" also shown in Figure 6; the locations were measured using GPS. The first position, "B," was 17 meters away from the static node; we then moved the node at discrete intervals and kept it at that location for approximately 5 minutes. We continued this until the two nodes could no

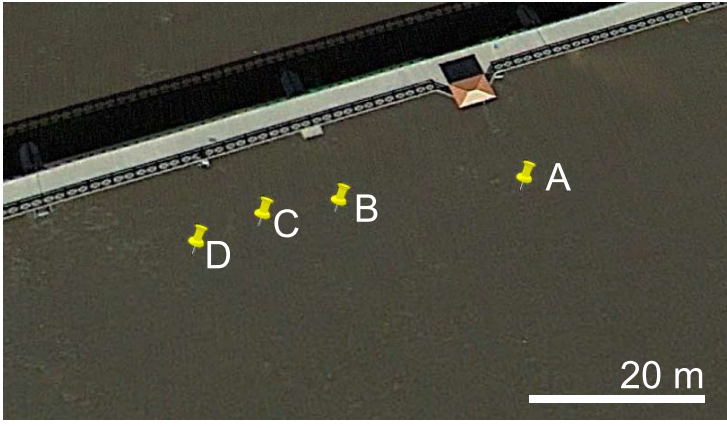

Fig. 6. Water node to water node test configuration. Figure courtesy of Google Earth.

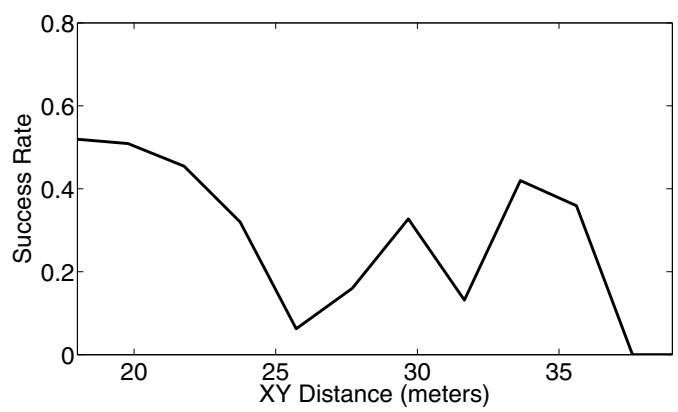

Fig. 7. Radio communication packet-success rates between two water nodes on the surface of the water at different distances.

longer communicate.

2) Test Results: Figure 7 shows the results for the water node to water node radio communication. The two significant drops in success rate at 25.8 and 32 meters indicate locations with limited measurements; as the node moved through the location on the way to the next fixed location, a small number of packets were sent but not received.

As the figure shows, the nodes already experience heavy packet loss at 17 meters, with a success rate of only $50 \%$. While we see significant variation over the full distance, it never improves above $50 \%$ and, on average, degrades in performance. Once the distance between nodes increases past 37 meters, the nodes can no longer communicate. This experimental data fits with the theoretically predicted results that communication along the same altitude will perform significantly worse.

\section{Results Summary and Analysis}

We now analyze the combined results to understand possible topologies and configurations when using an UAV with an underwater sensor network.

Given the limited node-to-node ranges, we should use an UAV within the system to expand the coverage area. Depending on the UAV flight altitude, we see a range of successes. Table I summarizes the best distance between the UAV and surfaced nodes for given altitudes as well as the success rate at that distance. For example, if the UAV flies at an altitude of 43 meters, its flight plan needs to pass within 212 meters of each node to achieve $75 \%$ packet-success rate. If instead, the UAV flies lower at 14 meters, it needs to pass within 137 meters to achieve $70 \%$ packet-success rate. 


\begin{tabular}{|l|l|l|}
\hline Altitude (m) & Best XY Distance (m) & Success-Rate Threshold \\
\hline Water surface & $0-34$ & $40 \%$ \\
\hline 6 & $0-87$ & $60 \%$ \\
\hline 14 & $0-137$ & $70 \%$ \\
\hline 25 & $0-149$ & $75 \%$ \\
\hline 38 & $0-174$ & $94 \%$ \\
\hline 43 & $0-212$ & $75 \%$ \\
\hline 49 & $0-237$ & $10 \%$ \\
\hline $50+$ & none & $0 \%$ \\
\hline
\end{tabular}

TABLE I

BEST RADIO COMMUNICATION RANGES: WATER SURFACE TO UAV

\begin{tabular}{|l|l|l|}
\hline Internode communication & $\begin{array}{l}\text { Best UAV Alti- } \\
\text { tudes }(\mathbf{m})\end{array}$ & $\begin{array}{l}\text { Best Node Spac- } \\
\text { ing (m) }\end{array}$ \\
\hline Yes & $6-43$ & 34 \\
\hline No & 43 & 424 \\
\hline
\end{tabular}

TABLE II

BEST UAV ALTITUDES AND BEST NODE SPACING FOR MAXIMIZING NETWORK COVERAGE

With this information, we can analyze the options for maximizing communication success while also maximizing network coverage area. Table II outlines the communication parameters to achieve this for two different network configurations. The first configuration requires the underwater nodes to reach via radio at least one other node such that internode communication exists in the system. Relaxing the communication requirement, the second network configuration considers a system where the underwater nodes do not need to communicate while on the surface; each node is out of radio range of each other.

Table III describes how these results relate to possible coverage areas of the network. For a network of 25 nodes, requiring internode communication allows the network to cover an area of $0.029 \mathrm{~km}^{2}$; relaxing that requirement allows coverage of $4.494 \mathrm{~km}^{2}$. Overall, adding an UAV to the system allows the network coverage to expand by $154 \%$, a significant increase that expands the utility of such systems.

Theoretically, we expected to see an attenuation of approximately $-100 \mathrm{~dB}$ at a 34 meter distance node to node. However, for the UAV, at the maximum distance of 212 meters and an altitude of 43 meters, the theoretical attenuation is only $-85 \mathrm{~dB}$. This is a significant difference that shows that signal quality in the air can be dramatically better than along the surface.

\section{ENERGY}

After exploring the communication aspects of the system, we now examine the energy. We first identify experimentally measured parameters that characterize the energy and then define an energy model to explore tradeoffs between network size, distance between nodes, and other parameters. With this model, we explore the impact of these parameters.

\section{A. Energy Characterization}

To understand the energy use of the combined UAV and sensor network system, we measure key parameters needed for characterization. Table IV outlines these parameters for the UAV, the 802.15.4 radio, and the AquaNode sensor node.

We measured the UAV data through flight tests and postflight analysis. These measurements indicate the best flying

\begin{tabular}{|l|l|l|}
\hline & \multicolumn{2}{|c|}{ Area of Coverage (Kilometers ${ }^{2}$ ) } \\
\hline $\begin{array}{l}\text { Network Configura- } \\
\text { tion }\end{array}$ & $\begin{array}{l}\text { Internode Commu- } \\
\text { nication }\end{array}$ & $\begin{array}{l}\text { No Internode Com- } \\
\text { munication }\end{array}$ \\
\hline 25 Nodes & 0.029 & 4.494 \\
\hline 64 Nodes & 0.074 & 11.506 \\
\hline 100 Nodes & 0.116 & 17.978 \\
\hline
\end{tabular}

TABLE III

MAXIMUM AREA COVERED BY SPECIFIED NETWORK

\begin{tabular}{|l|l|}
\hline Energy Parameters & Value \\
\hline UAV Power at 10m/s (travel_power) & 115 Watts \\
\hline UAV Hover Power $($ hover_power $)$ & 92.3 Watts \\
\hline UAV Travel Distance Per UAV Battery & 7300 Meters \\
\hline UAV Battery Capacity & 83900 Joules \\
\hline \hline Radio Transmit Power $($ max_transmit) & $63 \mathrm{mWatts}$ \\
\hline Radio Max Physical Data-rate & $57600 \mathrm{Bits} / \mathrm{Sec}$ \\
\hline $\begin{array}{l}\text { Radio Max Transmit Data-rate } \\
\text { (radio_true_data_rate) }\end{array}$ & $4800 \mathrm{Bits} / \mathrm{Sec}$ \\
\hline \hline AquaNode Battery Capacity & 172800 Joules \\
\hline AquaNode Depth Adjustment Power & 15 Joules/Meter \\
\hline AquaNode Acoustic Receive & 0.063 Joules/Bit \\
\hline AquaNode Acoustic Transmit & 0.1136 Joules/Bit \\
\hline AquaNode Flash Memory & 512000 Bits \\
\hline
\end{tabular}

TABLE IV

MEASURED ENERGY CHARACTERIZATION PARAMETERS

speed of the UAV, the energy used to travel at that speed, and the power used when hovering. In addition, we determine, for a given battery capacity, the UAV travel distance on a single battery. The radio_true_data_rate parameter is based on lab measurements. The radios operate at the maximum transmit power (max_transmit $=63 \mathrm{~mW}$ ) in order to maximize range. Although the physical layer transmits at 57600 bits per second (bps), the ZigBee radios transmit each packet multiple times to increase the likelihood of successful reception, and our experiments showed a true transmission data rate of 4800 bps.

\section{B. Energy Model}

Given these parameters, we now model the energy system of the UAV in order to understand trade-offs. For the UAV to communicate with the underwater sensor network, the UAV needs to travel to and traverse the network, hover above nodes, transmit and receive data via radio, and then fly back. We assume that no communication with the sensor network occurs while the UAV is traveling. In this scenario, the total energy expended by the UAV is:

$$
E_{U A V}=E_{\text {travel }}+E_{\text {hover }}+E_{\text {radio }}
$$

from each node, while leaving the transmission data rate fixed at the experimentally measured value.

\section{Energy Analysis}

Given the energy model, we now explore the ramifications of the parameters on the system topology. The key variables defining the energy of the UAV are: number of nodes in the sensor network, distance between nodes in the sensor network, distance out to the sensor network, and time spent hovering to collect data at each node.

We examine the impact of each of these parameters on the UAV energy; skewing one of them while fixing the rest. Our fixed default parameters are a network size of 100 nodes, 

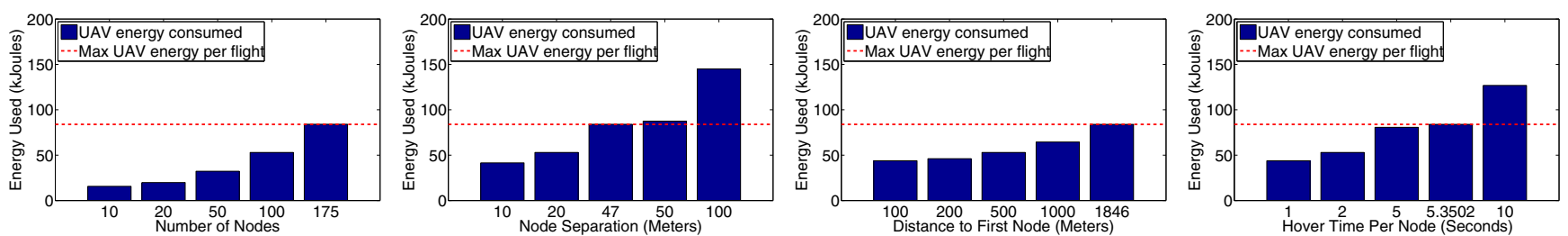

Fig. 8. Total UAV energy while varying parameters: (a) Network size, (b) Node spacing, (c) Distance to network, and (d) Hover time

a node spacing of 20 meters between nodes, a network distance of 500 meters from the UAV base, and a hover time of 2 seconds (which is 9.6 kbits of data transferred). Figures 8(a) through 8 (d) outline the results. Each bar graph represents a single parameter change; the red dotted line in each represents the maximum energy of the UAV.

Figure 8(a) describes the impact of the number of nodes in the network; for this parameter, at network sizes of 175 nodes or greater a single UAV does not have enough energy to collect all the data in 1 trip. Figure 8(b) shows the results varying node spacing. With this configuration, a single UAV can only handle 47 meter node spacing or less. Next, Figure 8(c) skews the distance of the network from the UAV base station while fixing the remaining parameters. Here, the UAV can only support a system that is within 1846 meters. Finally, Figure 8(d) varies the hover time from 1, 2, 5, and 10 seconds (or data transmission per node of 4.8, 9.6, 24, and $48 \mathrm{kbits}$ ). The best a single UAV can achieve is 5.3 seconds of hover, which is equivalent to a data transfer of 25681 bits per node.

Overall, the key factors affecting the overall energy level are the separation between the nodes and the hover time the UAV spends at each node seen in the greater energy usage over the increased values. These results do not consider communication; we can now examine the combined effects.

\section{Overall AnAlysis AND Algorithms}

After examining communication and energy separately, we can now explore algorithms for systems combining underwater sensor networks and UAVs. The communication ranges and ensuring the UAV returns safely to the home base are the two key limiting factors that constrain the system topology and installation. Given these two constraints plus an area to cover, we examine the number of nodes in the network, the possible distance of the network from the UAV base station, and the amount of time the UAV visits each node (which defines the amount of data it can collect).

We use our communication and energy information to develop Algorithm 1. This algorithm uses distance from the UAV base $\left(D_{n}\right)$, amount of data to communicate $\left(t_{\text {hover }}\right)$, and the spacing policy to determine the number of nodes a single UAV can support ( $L_{\text {spacing }}$ or Area). If we choose to define the spacing between nodes based on the configurations from Table II (either spacing at $34 \mathrm{~m}$ or $424 \mathrm{~m}$ ), the algorithm provides the network size and the area covered. If we choose to provide the area to cover, the algorithm computes the number of nodes and spacing between the nodes.

We first explore the internode communication options where, in addition to collecting data with the UAV, we want the sensor network to communicate using radios, limiting the node spacing to a maximum of 34 meters. Figure 9(a) shows the results of this spacing as we limit the parameters to ensure the UAV has sufficient energy to return to base. Within this limitation, the maximum network size possible is 160 nodes; any more than that and the system no longer functions (either the UAV has insufficient energy, the network is at the UAV base, or the UAV cannot visit the nodes for even a second). For a network of only 20 nodes, this indicates that the closest node can be 2,500 meters from the UAV base and the UAV can collect 10 seconds of data from each node. However, for a network of 160 nodes, the closest node of the network needs to be within 500 meters and the UAV can only collect 9,600 bits of data from each node.

If we relax the internode communication requirement, we can space the nodes at 424 meters apart as determined in Section V-C. Figure 9(b) shows the results of this spacing. Here the data collection time and range from the UAV base remain the same, but the number of nodes the network can contain reduces to a minimum of 4 and a maximum of 15 . Placing the network 400 meters from the UAV base and collecting data for only 1 second, the UAV can support a network of 15 nodes. With a minimum of 4 nodes, the network can exist 2500 meters away and the UAV can collect data for 10 seconds per node.

The above results assume the goal is maximizing area covered while minimizing the likelihood of losing the UAV. We instead could define the goal as maximizing the density of nodes we can place within a fixed area, which allows the communication spacing to vary between 34 and 424 meters. Figure 9(c) shows the possible network sizes in a $2 \mathrm{~km}^{2}$ area that a single UAV can handle in a single trip. Surprisingly, for a network that is 2500 meters from the UAV base where the UAV collects 10 seconds of data per node, the UAV cannot support any network size and still return to the UAV base. However, as long as the network is within 500 meters from the UAV base and the UAV collects 1 second of data per node, the network can contain 21 nodes.

Overall, Algorithm 1 outlines the possible options for the network configuration and topology based on whether the key factor is the maximum spacing between nodes or a specific area. Without consideration of area the largest network size the system can support is 160 nodes at 34 meter spacing, the largest area for coverage while still allowing the UAV to provide communication connection $2.7 \mathrm{~km}^{2}$, and a sensor network covering a $2 \mathrm{~km}^{2}$ area cannot exist at over 2500 meters from the UAV base.

\section{CONCLUSION}

We measured communication and energy characteristics of a system consisting of an UAV and an underwater sensor network with depth adjustment capabilities. Given the results of 

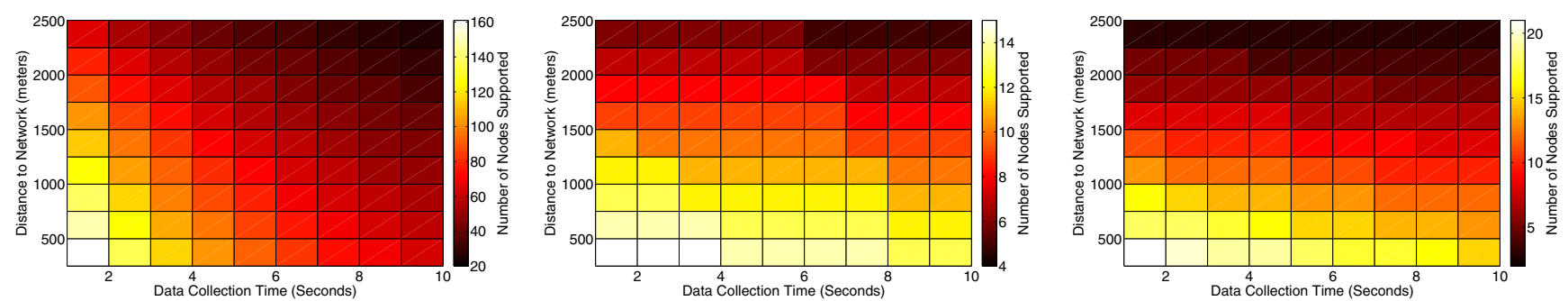

Fig. 9. Network sizes a single UAV can support for: (a) 34 m node spacing, (b) 424 m node spacing, and (c) a $2 \mathrm{~km}^{2}$ area. $^{2}$

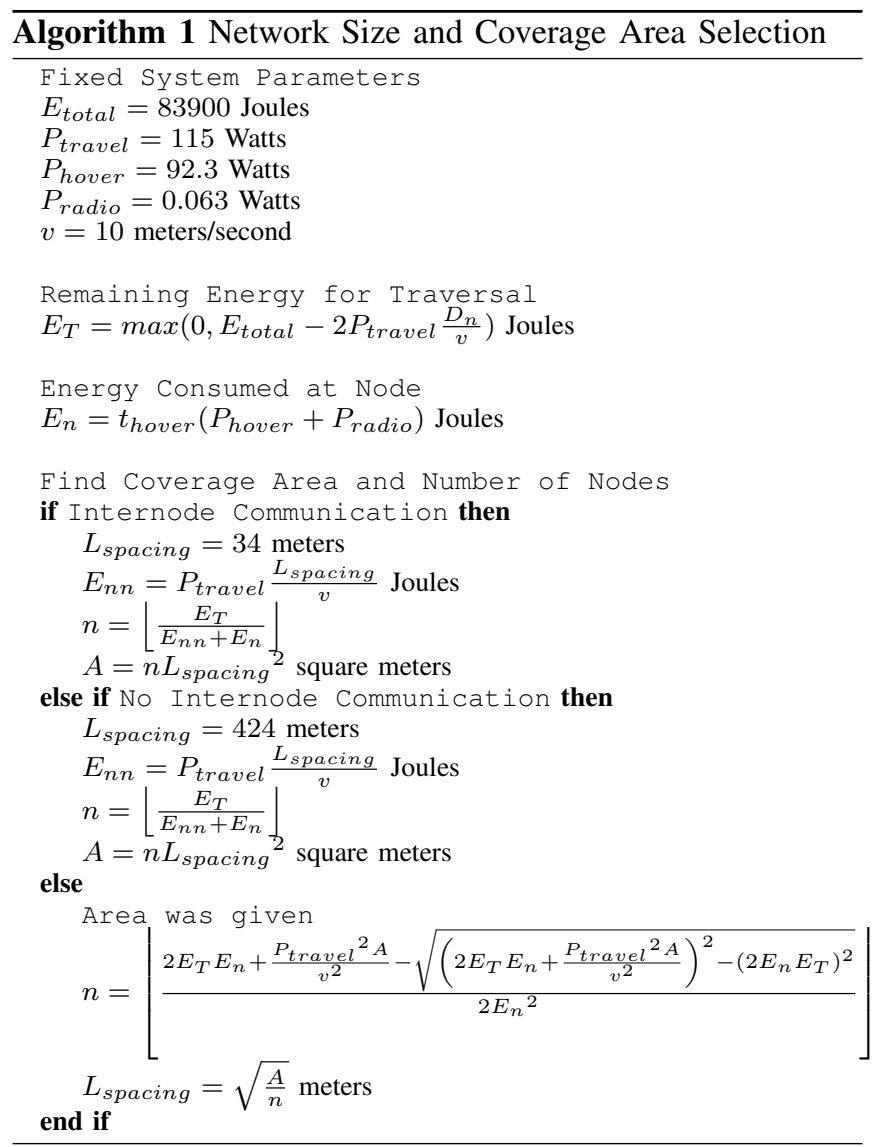

the communication experiments, we determined that adding an UAV allows the network area coverage to increase by $154 \%$. The energy characteristics show linear relationships between the four key parameters: network size, distance to network from the UAV base, node spacing, and amount of time UAV hovers at each node. Combining both results provides an algorithm that determines the network size in order to maximize network lifetime and spatial coverage.

Moving forward with these results, we plan to explore trade-offs between operational behaviors over time of the system (UAV visiting patterns, trajectory optimization, and other aspects). We plan to investigate the effects and improvements of incorporating expected signal strength via the theoretical model on path-planning algorithms with respect to packet reception rate and power use. We also plan to incorporate the acoustic communication patterns and data movements throughout the system to determine policies on how nodes share data, collect data, and control sensing behaviors as well as dynamic conditions such as weather and surface distortions.

\section{REFERENCES}

[1] S. K. Teh, L. Mejias, P. Corke, and W. Hu, "Experiments in integrating autonomous uninhabited aerial vehicles (uavs) and wireless sensor networks," in Australasian Conference on Robotics and Automation (ACRA). The Australian Robotics and Automation Association Inc., 2008.

[2] J. T. Isaacs, S. Venkateswaran, J. Hespanha, U. Madhow, J. Burman, and T. Pham, "Multiple event localization in a sparse acoustic sensor network using uavs as data mules," in Globecom Workshops (GC Wkshps). IEEE, 2012, pp. 1562-1567.

[3] D. J. Klein, J. Schweikl, J. T. Isaacs, and J. P. Hespanha, "On uav routing protocols for sparse sensor data exfiltration," in American Control Conference (ACC). IEEE, 2010, pp. 6494-6500.

[4] M. Todd, D. Mascarenas, E. Flynn, T. Rosing, B. Lee, D. Musiani, S. Dasgupta, S. Kpotufe, D. Hsu, R. Gupta, G. Park, T. Overly, M. Nothnagel, and C. Farrar, "A different approach to sensor networking for shm: Remote powering and interrogation with unmanned aerial vehicles," in Proceedings of the 6th International workshop on Structural Health Monitoring., 2007.

[5] R. Sugihara and R. K. Gupta, "Improving the data delivery latency in sensor networks with controlled mobility," in Distributed Computing in Sensor Systems, ser. Lecture Notes in Computer Science. Springer, 2008, vol. 5067, pp. 386-399.

[6] _ _ "Optimizing energy-latency trade-off in sensor networks with controlled mobility," in International Conference on Computer Communications (INFOCOM). IEEE, 2009, pp. 2566-2570.

[7] — "Speed control and scheduling of data mules in sensor networks," ACM Transactions on Sensor Networks (TOSN), vol. 7, no. 1, p. $4,2010$.

[8] - "Optimal speed control of mobile node for data collection in sensor networks," in Mobile Computing, vol. 9, no. 1. IEEE, 2010, pp. 127-139.

[9] I. Vasilescu, K. Kotay, D. Rus, M. Dunbabin, and P. Corke, "Data collection, storage, and retrieval with an underwater sensor network," in Proceedings of the 3rd international conference on Embedded networked sensor systems. ACM, 2005, pp. 154-165.

[10] M. Doniec, C. Detweiler, I. Vasilescu, M. Chitre, M. Hoffmann-Kuhnt, and D. Rus, "AquaOptical: A lightweight device for high-rate longrange underwater point-to-point communication," Marine Technology Society Journal, pp. 55-65, Aug. 2010.

[11] K. Li, C.-C. Shen, and G. Chen, "Energy-constrained bi-objective data muling in underwater wireless sensor networks," in Mobile Adhoc and Sensor Systems (MASS). IEEE, 2010, pp. 332-341.

[12] C. Detweiler, M. Doniec, I. Vasilescu, and D. Rus, "Autonomous depth adjustment for underwater sensor networks: Design and applications," IEEE Transactions on Mechatronics, vol. 17, no. 1, pp. 16-24, 2012.

[13] S. Jiang and S. Georgakopoulos, "Electromagnetic wave propagation into fresh water," Journal of Electromagnetic Analysis and Applications, vol. 3, no. 7, pp. 261-266, 2011.

[14] T. Rappaport, Wireless Communications: Principles and Practice, 2nd ed. Upper Saddle River, NJ, USA: Prentice Hall PTR, 2001.

[15] "Radio propagation, multipath and diversity antennas," http://www.allenhollister.com/allen/files/radiopropagation.pdf, accessed: 2014-10-01.

[16] K. Bullington, "Radio propagation at frequencies above 30 megacycles," Proceedings of the IRE, vol. 35, no. 10, pp. 1122-1136, Oct 